\title{
REAL-WORLD SORTING OF RHIC SUPERCONDUCTING MAGNETS*
}

\author{
$\underline{\text { J. Wei }}^{\dagger}$, R. Gupta, M. Harrison, A. Jain, S. Peggs, P. Thompson, D. Trbojevic, P. Wanderer \\ Brookhaven National Laboratory, Upton, NY 11973, USA
}

\section{Abstract}

During the seven-year construction of the Relativistic Heavy Ion Collider (RHIC), more than 1700 superconducting dipoles, quadrupoles, sextupoles, and multi-layer correctors have been constructed and installed. These magnets have been sorted at several production stages to optimize their performance and reliability. For arc magnets, priorities have been put first on quench performance and operational risk minimization, second on field transfer function and other first-order quantities, and finally on nonlinear field errors which were painstakingly optimized at design. For Interaction-Region (IR) magnets, sorting is applied to select the best possible combination of magnets for the low- $\beta^{*}$ interaction points (IP). This paper summarizes the history of this real-world sorting process.

\section{INTRODUCTION}

The RHIC magnet system consists primarily of superconducting dipole, quadrupole, sextupole and corrector magnets for guiding, focusing, and correcting the countercirculating ion beams into the design orbits in the regular arcs of the machine lattice. A large complement of special superconducting magnets is also required for steering the beams into collisions at the six interaction regions (IR) where the ion beams interact. During the seven-year construction cycle, more than 1700 superconducting magnets have been constructed, measured, installed and tested. In order to optimize the performance of these magnets, sorting has been applied whenever possible.

For a majority of the arc magnets, priorities have been put first on quench performance and operational risk minimization and second on field transfer function and other first-order quantities. Since nonlinear field errors were painstakingly optimized at design, and their sorting priority was low. For IR magnets, sorting was applied to select the best possible combination of magnets for 2 out of 6 IRs where $\beta^{*}$ will be lowered to 1 meter for high luminosity experiments. In order to minimize the relative misalignment between magnets in a common cryostat, sorting was also applied both before and after cryostat assembly. In contrast to an idealized magnet sorting, sorting in a real world is often constrained by the assembly and installation schedule, available storage space, etc. This paper summarizes the history of this real-world sorting process. In Section 2, we review the overall procedure of magnet analysis, acceptance, and sorting. In Sections 3 and 4, we summarize the actual sorting experience for arc and IR magnets.

* Work performed under the auspices of the US Department of Energy.

'Email: weil @bnl.gov

\section{MEASUREMENT DATA ANALYSIS}

Besides reaching fields with substantial margins above the required range, all of the RHIC magnets must meet stringent requirements for field quality, reproducibility, and long-term reliability. In order to fulfill this goal, a committee of magnet division and RHIC accelerator physics personnel jointly reviewed the field quality, quench test performance, survey and other engineering aspects of the magnets. After individual magnet elements (coldmasses) are measured and tested, the magnetic field quality data. including transfer function, field angle, multipole harmonics, magnetic center offsets, etc. at all the test currents, [1] are recorded along with the warm mechanical survey measurements of the fiducial positions, sagitta, mechanical length and field angle. The data are transferred from the magnet division into the RHIC SYBASE database, and then analyzed by studying trends, comparing with the expected values, and evaluating the deviation from the mean using the computer program MAGSTAT [2]. As shown in Fig. 1, after their review and acceptance, magnets contained in their own cryostats (e.g. arc dipoles) are sorted for their candidate installation locations. Magnets belonging to a common cryostat assembly go through a second stage of

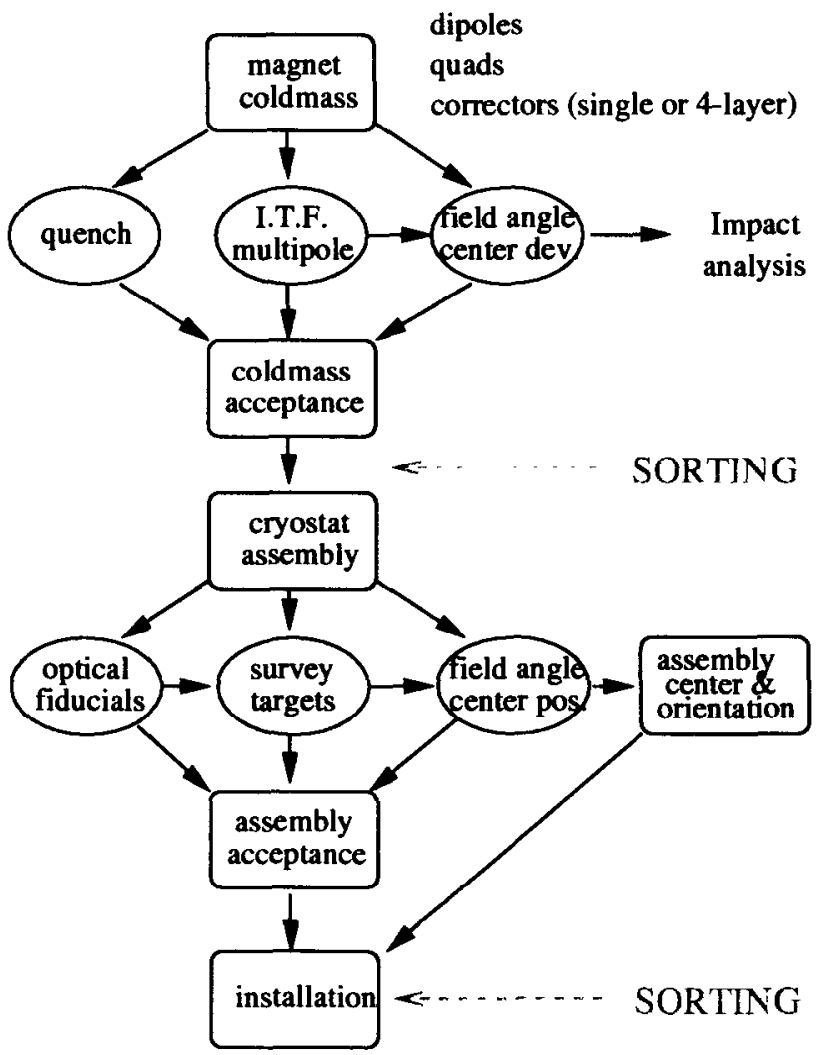

Figure 1: Magnet acceptance and sorting procedure. 
review, acceptance and sorting. At this stage, the assembly is surveyed with either colloidal-cell optical or stationarycoil pick-up (antenna) techniques to locate the magnetic centers of the components relative to the cold mass fiducials and the externally accessible cryostat fiducials. This survey data is transferred into the database and analyzed using the computer program SURVSTAT [3]. Based on a second-round review and balance of both coldmass and assembly data, the assemblies are sorted for final installation.

\section{ARC REGION MAGNET SORTING}

RHIC arc dipoles, quadrupoles and sextupoles are industrybuilt magnets. Despite close communication and detailed quality assurance procedures, unexpected changes in the manufacturing process still occurred. Magnet acceptance review and the subsequent sorting played an essential role in optimizing the final performance.

\subsection{Arc Dipoles}

During the acceptance, a drop in the integral transfer function (ITF) of about $0.1 \%$ was noticed and traced to the narrower width of the phenolic insulator used between the coil and the iron. Although the problem was corrected, about 20 magnets were affected. These magnets were sorted along with all subsequent dipole magnets. The sorting procedure was based on the strength minimization of dipole correctors required to compensate for the variation in the integral transfer function. With sorting, the maximum current required for such compensation was decreased from $12 \mathrm{~A}$ to about $3 \mathrm{~A}$.

The dominant multipoles of the dipole magnets are $b_{2}$ (normal sextupole) and $b_{4}$ (normal decapole) resulting from the dipole symmetry of the magnets, and $a_{1}$ (skew quadrupole) resulting from the asymmetric vertical placement of the magnet cold mass in the cryostat. Due to the relatively high injection energy and the small diameter of the coil filaments, the persistent current effects are small. Magnet design has minimized $b_{2}$ and $b_{4}$ for both injection and storage currents by optimizing the cross-sections of the coil and the yoke taking into account the persistent current and saturation effects. The minimization of $a_{1}$ is achieved by sorting the yoke weight during the assembly process so that the lower half yoke is heavier than the upper half.

Among the eight dipoles allocated as spare magnets, five of them have off-normal skew quadrupole component $\left(a_{1}\right.$ up to -5.9 units [4]), some caused by a known coil size mismatch; one has an excessive twist ( $2.5 \mathrm{mr}$ standard deviation in body field angle) along the magnet body, and one has low transfer function at high fields.

\subsection{Arc Quadrupoles}

At the early stage of industrial manufacture, midplane shims were incorrectly changed on 5 quadrupoles, resulting in a $b_{3}$ of about -6 units. These magnets were sorted and distributed among the two rings to minimize their effects.

The dominant multipoles of the quadrupoles are $b_{5}$ and $a_{5}$ resulting from the quadrupole symmetry of the coil and the end configuration, and $b_{3}$ resulting from the asymmetry between the horizontal and vertical planes. $b_{3}$ was compensated in the design by making the coil to midplane gap appropriately asymmetric, while $b_{5}$ was reduced by compensating the body with the ends of the magnet.

Among the eight quadrupoles allocated as spare magnets, four of them are of concern with off-normal coil size or low collaring pressure, some resulting in large $a_{2}$; two have excessive $b_{2}$ ( -5 units); one has an engineering repair.

\subsection{Arc Sextupoles}

In general, the performance of the sextupole magnets exceeded the design goal. However, the epoxy contained in about $\mathbf{4 2}$ magnet coils is significantly weaker than normal. Consequently, the average quench currents (about $170 \mathrm{~A}$ ) of these magnets, although exceeding the design operating current $(100 \mathrm{~A})$, are lower than the average of the regular magnets (above $200 \mathrm{~A}$ ). To minimize possible long-term effects, these magnets have been sorted and allocated to the focusing locations around the two rings where the required strength of the sextupoles for chromaticity correction is about $50 \%$ of that at the defocusing locations.

\subsection{Anc Trim Quadrupoles}

Trim quadrupoles all have minimum quench currents above $200 \mathrm{~A}$, well exceeding the design operating current of $100 \mathrm{~A}$. One trim quadrupole coldmass was designated as a spare due to rust on the yoke caused by rain damage.

\subsection{Arc Correctors}

All of the correctors, either single-layer or four-layer, were built in-house and cold tested. After initial training, all the magnets quench above the design operating current of $50 \mathrm{~A}$. Since the dipole corrector layers are all powered individually, the variation in the integral transfer function (typically $1 \% \mathrm{rms}$ ) is of little concern. Correctors with layers of excessive field angle deviation (up to $\sim 20 \mathrm{mr}$ ) or erratic quench training were selected as spare magnets.

\subsection{Arc CQS Assembly}

Arc corrector, quadrupole, and sextupole magnets were welded into a single "CQS" assembly. The CQS assembly also includes a beam position monitor and (for some) a recooler. The CQS components need to be aligned with each other so that their magnetic centers are on a straight line. It was found in the early stage of installation that "Springs" (made of G-10 plastic) needed to be installed or refitted in the support posts, confining the coldmass transversely while allowing free longitudinal motion. Special welding stripes were applied to the CQS shell to align the magnetic centers of the individual coldmasses for assemblies that exceeded a tolerance of $0.25 \mathrm{~mm}$. Subsequently, the welding sequence is carefully choreographed to balance "curling" distortions against each other.

Correctors with large misalignments can generate serious feed-down harmonics. Two early CQSs with corrector offsets larger than $2 \mathrm{~mm}$ have been removed from the tunnel, and were later corrected. 


\section{IR MAGNET SORTING}

The IR triplet cryostat contains two dipoles, six quadrupoles, and six four-layer corrector packages of the two rings. Field imperfection of the IR magnets limits the machine performance at collision when $\beta^{*}$ is squeezed. Among the 6 interaction points, 2 of them are planned to run at a low $\beta^{*}$ of $1 \mathrm{~m}$. Most sorting efforts have been to select the best IR magnets for these 2 "golden" IPs.

\subsection{IR Dipoles}

In general, two IR dipoles, one on each side of the IP, are powered by the same shunt power supply. Sorting has been performed to pair dipoles of similar transfer function to the same IP. Two dipoles with off-normal transfer function are assigned to special locations where individual shunt supplies exist. Early magnets with imperfect field quality (large $b_{2}$ ) were assigned to non-golden region. Since the outstanding random error is $a_{1}$, magnets of similar $a_{1}$ are sorted to the opposite side of the same IP to minimize their action kicks [5]. One dipole is designated as a spare due to erratic quench performance.

\subsection{IR Quadrupoles}

The manufacturing sequence of $I R$ quadrupoles follows the level of required performance, starting with the less-critical Q1. Several iterations were made on the magnet cross section to optimize the field quality. Application of tuning shims is also practiced at this stage.

In one Q2 quadrupole, an excessive amount of axial variation in multipole errors was found (change of 15 units of $a_{2}$ ) and suspected to be due to cracked insulators. The quench performance, though adequate, was lower than average. Efforts were made at the last stage of installation to replace this "golden candidate" with a "spare candidate".

Due to lack of time for cryogenic testing, 11 out of 72 IR quadrupoles were measured only at room temperature. Because of imperfect correlation between the warm and cold measurements, the field quality of these magnets is less well known than the field quality of magnets which have been cold tested. Since this information is the critical base for IR correction, these magnets were sorted to "nongolden" IRs. Spare magnets were mostly selected based on off-normal multipole errors. One quadrupole with a partially inserted shim was first allocated as a spare but later installed to meet schedule requirements for the first sextant test.

\subsection{IR Correctors}

Sorting on IR correctors was performed along with the quadrupoles before their attachment to minimize the relative magnetic center offset and field angle. After sorting, for CQ combinations with excessive relative offset and roll, shimming adjustment were made before welding of the assembly.

\subsection{IR CQ Assembly}

IR correctors were welded to IR quadrupoles to form CQ assemblies. At a later stage of IR CQ assembly, electric
Table 1: Summary of RHIC magnet sorting ( $n=1$ is quadrupole).

\begin{tabular}{|c|c|c|}
\hline Magnet & $\begin{array}{l}\text { Number } \\
\text { (used+spare) }\end{array}$ & Sorted quantity \\
\hline Arc dipole & $288+8$ & $\begin{array}{l}\text { ITF, yoke weight }\left(a_{1}\right) \\
\text { twist, } b_{2}\end{array}$ \\
\hline Arc quad. & $372+8$ & $\begin{array}{l}\text { coil size, midplane shim size } \\
\text { collaring pressure } \\
\text { coil saddle crack repair, } b_{2}\end{array}$ \\
\hline $\begin{array}{l}\text { Arc sext. } \\
\text { Arc corr. }\end{array}$ & $\begin{array}{l}288+12 \\
420+10\end{array}$ & $\begin{array}{l}\text { epoxy level (quench) } \\
\text { quench, field angle }\end{array}$ \\
\hline D5I & $12+1$ & vacuum vessel straightness \\
\hline D50 & $12+1$ & vacuum vessel straightness \\
\hline D96 & $48+1$ & \\
\hline Trim quad. & $72+6$ & rust on yoke \\
\hline CQS & $282+8$ & corrector offset \\
\hline CQT & $72+6$ & \\
\hline $\mathrm{CQ}$ & $60+2$ & \\
\hline \multicolumn{3}{|c|}{ Interaction region magnets: } \\
\hline IR dipole & $24+2$ & quench, ITF, $b_{2}, a_{1}$ \\
\hline IR quad. & $72+6$ & $\begin{array}{l}\text { data availability (schedule) } \\
\text { axial variation of } a_{2} \\
\text { partial shim, multipoles }\end{array}$ \\
\hline IR corr. & $72+6$ & center offset, roll \\
\hline DX dipole & $12+1$ & \\
\hline $\mathbf{I R C Q}$ & $72+6$ & potential corrector shorts \\
\hline Total & $1692+65$ & \\
\hline
\end{tabular}

shorts were found at the octupole leads of IR correctors precipitated by a routing misdesign. Rework was done on all the correctors which were still in coldmass state. For correctors designated as "golden" and yet with their end plates already welded on, their end plates were removed to allow a complete rework. About $8 \%$ of the $C Q$ assemblies fully installed in the machine were not reworked, and their chance of octupole layer malfunction is less than $10 \%$.

\subsection{Separating Dipoles DX}

After a design iteration based on the prototype magnet, the field errors $\left(b_{2}, b_{4}\right)$ of these large-bore $(18 \mathrm{~cm}$ coil diameter) dipoles were greatly reduced and are well within the capability of IR correction [6].

We thank members of the Magnet Acceptance Committee for their contribution.

\section{REFERENCES}

[1] P. Wanderer, CERN 98-05 (1998) p. 273.

[2] J. Wei, G. Ganetis, R. Gupta, M. Harrison, M. Hemmer, A. Jain, F. Karl, S. Peggs, S. Tepikian, P.A. Thompson, D. Trbojevic, and P. Wanderer, EPAC96 (1996) p. 2222.

[3] J. Wei, R. C. Gupta, A. Jain, S. G. Peggs, C. G. Trahem, D. Trbojevic, and P. Wanderer, PAC95 (1995) p. 461.

[4] Multipole errors are normalized to $10^{-4}$ of their integral strength at a reference radius of about $2 / 3$ coil radius. $n=1$ is quadrupole.

[5] J. Wei, W. Fischer, V.Ptitsin, R. Ostojic, J.Strait, PAC99 (1999).

[6] A. Jain, PAC99 (1999). 